\title{
Effects of discriminability and irrelevant information on absolute judgments'
}

BEN B. MORGAN, JR., AND EARL A. ALLUISI

UNIVERSITY OF LOUISVILLE

The effects of irrelevant information $(0,1,2$, or 3 bits/ stimulus) on absolute judgments of size were measured at different levels of discrimination difficulty. The stimuli were 7 small circular spots of light of different sizes, selected from an equal-discriminability scale, and arranged into 6 pairs that represented different levels of stimulus dissimilarity. Three of the pairs comprised a low-dissimilarity(LD) subgrouping in which different levels of dissimilarity and discriminability were confounded, whereas the remaining three pairs comprised a high-dissimilarity (HD) subgrouping in which different levels of dissimilarity occurred at a single high level of discriminability. Different colors were used to provide the four levels of irrelevant information; $240 \mathrm{Ss}$ (10 in each of the 24 experimental conditions) made absolute judgments of size under the classical procedure for the method of single stimuli. Separate analyses of errors, reaction times, and rates of information transmission indicated that irrelevant information had an increasingly detrimental effect on absolute judgments as discrimination difficulty was increased (in the LD subgroup), but essentially no effect where discriminations were easily made(in the $\mathrm{HD}$ subgroup).

The time required both for solving a conceptidentification problem (Archer et al, 1954) and for making visual (Gregg, 1954) and auditory (Montague, 1965) discriminations has been found to increase with increases in the amount of irrelevant information presented with the stimuli. On the other hand, the time required for making simple visual pattern discriminations has been found to be independent of the amount of irrelevant information presented (Archer, 1954).

This discrepancy in results has been explained partially on the basis of the way in which the irrelevant information is employed as an experimental variable (Hodge, 1959). That is to say, where the irrelevant information is used in such a way as to increase the likelihood of competing responses (e.g., where the information is sometimes relevant, but irrelevant at other times), it is likely to influence performance adversely. The detrimental effects of this "sometimes-relevant" irrelevant information on complex visual discriminations appears also to be a function of the difficulty of the discriminations involved; the more difficult the discrimination, the greater the detrimental effect of this kind of irrelevancy (Hodge, 1959).

Thus, it appears that information which is always irrelevant may (Gregg, 1954) or may not (Archer, 1954) affect both the amount of information transmitted in absolute judgments and the speed with which these judgments are made. On the other hand, information that is sometimes relevant can be expected to affect performance adversely on those occasions when it is irrelevant, and increases in the difficulty of the discriminations have been found to increase the adverse effects of the latter type of irrelevancy (Hodge, 1959). Furthermore, since Archer's (1954) task was easier than Gregg's (1954), there is a suggestion that the difficulty of the discrimination may also interact with the always-irrelevant type of irrelevant information.

The present study was designed to investigate the effects of irrelevant information (of the always-irrelevant type) on absolute judgments at different levels of discrimination difficulty.

\section{Method}

Four levels of irrelevant information $(0,1,2$, or $3 \mathrm{bits} / \mathrm{stimulus)}$ were combined factorially with six levels of stimulus dissimilarity to provide 24 different experimental conditions. Ten Ss were assigned at random to each of the conditions.

Stimuli. The stimuli were seven small circular spots of light of different sizes (ranging in diameter from $3 / 64$ to $15 / 64$ in.) selected from an equaldiscriminability (ED) function (Garner \& Hake, 1951) that had been constructed and validated previously (Alluisi \& Sidorsky, 1958). The stimuli were arranged into six pairs that represented six different levels of stimulus dissimilarity-a subset of the ten pairs previously employed in a study of choice time and stimulus dissimilarity (Thurmond \& Alluisi, 1963). The smallest spot of light (with an ED-scale value of 3.0) was used as the "smaller" stimulus in each of the six pairs. The "larger" stimuli were selected so as to provide stimulus dissimilarity (SD) values of $1,2,3$, 4,8 , and 12, as defined elsewhere (Thurmond \& Alluisi, 1963, pp. 327-328). It is important to note that although the six pairs of stimuli represented six different levels of dissimilarity, they did not necessarily represent six different levels of discriminability. It has been found under conditions of no irrelevant information that discriminability increases with SD values up to a "critical value" of 3.5 ; pairs of stimuli with SD values greater than 3.5 may differ in terms of dissimilarity, but they represent a single high level of discriminability (cf., Thurmond \& Alluisi, 1963). Thus, in the present study, three of the stimulus pairs (with SD values of 1,2 , and 3) comprise 
a low-dissimilarity (LD) subgrouping in which different levels of dissimilarity and discriminability are confounded, whereas the remaining three pairs (with SD values of 4,8 , and 12) comprise a high-dissimilarity (HD) subgrouping in which different levels of dissimilarity occur at a single high level of discriminability.

The four levels of irrelevant information were provided by use of ten different colors. The colors used were those described in a prior scaling as (1) dark blue, (2) light blue, (3) dark green, (4) light green, (5) gray, (6) yellow, (7) orange, (8) dark red, (9) light red, and (10) violet. The colors were produced by the use of specified sets of Wratten filters, glass enclosed in metal slide binders (see Muller et al, 1955, p. 13). Either 1 (color 5), 2 (colors 2 and 9), 4 (colors 2, 4, 6, and 8 ), or 8 (colors $1,2,3,5,6,7$, 9 , and 10) different colors were used to provide 0,1 , 2 , or $3 \mathrm{bits} / \mathrm{stimulus}$ of irrelevant information, respectively. That is to say, with no irrelevant information, all stimuli were of the same color-the color produced by use of the neutral-density filter; at the 1-bit/stimulus level of irrelevant information, colors 2 and 9 (light blue and light red) were randomly paired with each circle-of-light stimulus on different presentations, etc. The colors were chosen so as to provide "most discriminable" sets of 2, 4, or 8 colors.

Apparatus. The experiment was conducted in a 6-by$8 \mathrm{ft}$. experimental booth. The stimuli were displayed singly at the center of a 3-in. diameter circular display area of opal glass that was located at the center of a $36 \times 36$ in. plywood surface. A 1/2-in. diameter green jewel indicator light, which was used to provide a "ready" signal, was located $3-1 / 2$ in. below the center of the opal glass screen. The entire display surface was tilted 30 degrees from the vertical away from $S$, and its center was 30 in. above the floor level of the experimental booth.

Stimuli were formed by projecting light onto the opal glass screen through holes drilled in specially constructed slides of $1 / 16-i n$. thick opaque black plexiglas. The holes were accurately drilled; the position, circularity, and size (diameter) of each was verified with a microscope and hemocytometer. Each diameter employed was within 0.0008 in. of the value prescribed by the desired SD and ED-scale values (Thurmond \& Alluisi, 1963, pp. 327-328) and the previously published ED function (Alluisi \& Sidorsky, 1958 , p. 89).

The source for the stimulus light was a standard slide projector located approximately 27 in. behind the display surface. The beam of light was reduced by a lens stop that consisted of a metal disk with a 1/8-in. diameter hole in its center. A lightproof tube was attached to the projector and extended to the rear of the display surface to prevent stray light from falling on the rear of the display while stimulus slides were being positioned. For each stimulus presentation, the appropriate slide was positioned directly behind the opal glass so that its circular spot of light would appear on the center of the display surface; a slide holder and guide was constructed to permit rapid and exact positioning of the slides. The irrelevant color stimulus was superimposed on the circular spot of light by use of the appropriate color filter in a separate slide holder located between the light source and the stimulus slide. Other details of the apparatus are identical to those reported elsewhere (Thurmond \& Alluisi, 1963, p. 328).

Subjects. The $240 \mathrm{Ss}$ (10 in each of the 24 experimental conditions) were volunteers from introductory psychology classes at the University of Louisville. Five men and five women were assigned at random to each of the experimental groups. All Ss possessed normal or corrected $20 / 20$ visual acuity as measured with a Bausch and Lomb Ortho-Rater.

Procedure. Each $\mathrm{S}$ was tested individually. He was seated in the experimental booth facing the display surface, and viewed the stimuli binocularly from a distance of approximately $28 \mathrm{in}$. He was instructed to respond to the smaller stimulus by depressing a response key placed under his preferred hand, and to respond to the larger stimulus by depressing a response key placed under his nonpreferred hand. He was informed of the "ready" signal (the green indicator light that was lit during the 2-sec. interval preceding the stimulus presentation) and was shown the two stimuli with which he was to work. He was then given three practice trials followed by 96 experimental trials.

Presentation of the stimuli followed the classical procedure for the method of single stimuli. A trial consisted of the 2-sec. "ready" signal followed by the presentation of one of the two stimuli in the pair assigned to $S$, and by S's key-pressing type-b reaction. Immediately following S's response, $\mathrm{E}$ provided knowledge of results verbally. The stimuli were presented in a random order (different for each $\mathrm{S}$ ) with the restriction that each of the two stimuli in a given pair was presented an equal number of times during each session of 96 trials. The different colors, used to provide the several levels of irrelevant information, were assigned at random to the stimulus presentations with the restriction that each of the stimuli would have an equal number of pairings with each of the colors being used in a given condition. The stimulus presented, the response made, and S's RT (to the nearest $0.01 \mathrm{sec}_{\text {.) }}$ were recorded for each trial. Additional details of the methodology are given elsewhere (Morgan, 1965).

\section{Results}

Errors, RT, and rates of information transmission were separately analyzed in terms of the 4 by 6 (irrelevant information by stimulus dissimilarity) factorial design of the study. In addition, subgroups 


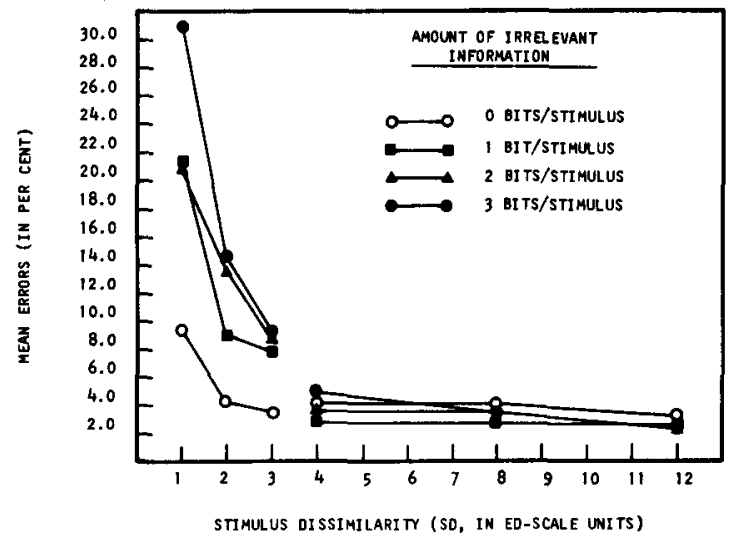

Fig. 1. Mean percentage of errors as a function of stimulus dissimilarity at four levels of irrelevant information.

were analyzed in terms of the $\mathrm{LD}$ and HD (low and high discriminability) groupings of the pairs of stimuli.

Errors. Mean errors (in percent) are shown in Fig. 1 as a function of stimulus dissimilarity (SD value, in ED-scale units) for each of the four levels of irrelevant information employed $(0,1,2$, and 3 bits/stimulus). It is apparent that errors drop off very rapidly with increases in dissimilarity between the SD values of 1 and 3 ; also, errors appear to be a direct function of the amount of irrelevant information presented within this range of SD values (the $L D$ subgroups). On the other hand, errors are relatively stable over the range of SD values from 4 to 12 (the HD subgroups), and appear also to be unaffected by the different levels of irrelevant information used.

An analysis of variance of the data of Fig. 1 indicated that the differences among the six levels of stimulus dissimilarity were statistically significant $(F=55.723$, $\mathrm{df}=5 / 216, \quad \mathrm{p}<.001$ ), as were also the differences among the four levels of irrelevant information $(F=11.182, \mathrm{df}=3 / 216, \mathrm{p}<.001)$, and the interaction of these two variables $(F=4.166, d f=15 / 216, p<.001)$. The general analysis was extended by use of two additional analyses of variance suggested by the prior findings (Thurmond \& Alluisi, 1963) that the LD and HD subgroups differ.

Within the LD subgroups, statistically significant differences were found among the three levels of stimulus dissimilarity $(F=33.372, \mathrm{df}=2 / 108, \mathrm{p}<.001)$, the four levels of irrelevant information $(F=13.770$, $\mathrm{df}=3 / 108, \mathrm{p}<.001$ ), and the interaction of the two variables $(F=2.134, \mathrm{df}=6 / 108, \mathrm{p}<.05)$. Within the HD subgroups, no statistically significant differences were obtained (only the dissimilarity variable produced an $F$-ratio greater than unity, and in that case $F=1.794$, $d f=2 / 108, p>.10)$. These results are essentially the same as those reported previously with no irrelevant information (Thurmond \& Alluisi, 1963, pp. 331-332).

Disjunctive RT. Each S's median RT to both stimuli in the pair with which he worked was computed. As was the case in the previous study (Thurmond \&
Alluisi, 1963, p. 333), the within-cell variances of the median RTs were not homogeneous, so a reciprocal transformation was applied and homogeneity of variance was obtained. The means of the reciprocals of the median RTs are shown in Fig. 2 as a function of stimulus dissimilarity for each of the irrelevantinformation conditions.

An analysis of variance of the data of Fig. 2 indicated that there were statistically significant differences among the six levels of stimulus dissimilarity ( $F=34.514, \mathrm{df}=5 / 216, p<.001)$ and among the four levels of irrelevant information $(F=3.444, \mathrm{df}=3 / 216$, $\mathrm{p}<.025)$. The interaction of these two variables was not statistically significant $(F=.813, d f=15 / 216, p>.10)$.

In the previous study, with no irrelevant information, it had been found that different slope constants were obtained with linear fits of the LD and HD data (Thurmond \& Alluisi, 1963, pp. 333-335). The data of Fig. 2 appear to follow the same pattern at the 0-bits/ stimulus level of irrelevant information; at the other levels of irrelevant information the pattern is not clear. In order to test for such differences between the $L D$ and $H D$ subgroupings, the analysis of variance was extended and eight regression analyses were computed-one at each level of irrelevant information in both the LD and the HD subgroupings. Summaries of these analyses are presented in Table 1.

Statistically significant linear regressions were obtained at each of the four levels of irrelevant information in both LD and HD subgroups. The deviations from linearity were not statistically significant in any case. The differences between the $L D$ and HD slope constants were statistically significant in the cases of the 0-bits/stimulus level of irrelevant information $(t=3.289, \mathrm{df}=56, \mathrm{p}<.001)$ and the 2-bits/ stimulus level $(t=2.415, \mathrm{df}=56, p<.05)$; they were not significant in the cases of the 1-bit/stimulus $(t=1.524$, $\mathrm{df}=56, \mathrm{p}>.05)$ or the 3 -bits $/$ stimulus levels $(t=1.213$, $\mathrm{df}=56, \mathrm{p}>.10$ ).

Rate of information transmission. The stimulus-



Fig. 2. Reciprocal of median reaction time as a function of stimulus dissimilarity at four levels of irrelevant information. 
Table 1. F-Ratios for Variances Due to Linear Regression and Deviations from Regression in both Low- and High-Discriminability Subgroups at Each of Four Levels of Irrelevant Information

\begin{tabular}{lcccccc} 
& \multicolumn{6}{c}{ Irrelevant-Information Conditions } \\
$\begin{array}{l}\text { Source of } \\
\text { Variation }\end{array}$ & df & 0 & 1 & 2 & 3 \\
\hline $\begin{array}{l}\text { LD Subgroups: } \\
\text { (SD Values < 3.5) }\end{array}$ & & & & & \\
$\begin{array}{l}\text { Linear Regression } \\
\text { Deviations }\end{array}$ & 1 & $12.461^{* *}$ & 3.093 & $10.307^{* *}$ & $4.207^{*}$ \\
Within Cells (MS) & 1 & 0.920 & 1.623 & 2.526 & 0.846 \\
Total (Sum of Squares) & 27 & $(0.106)$ & $(0.164)$ & $(0.096)$ & $(0.092)$ \\
\hline HD Subgroups: & & & & & \\
(SD Values $>3.5$ ) & & & & & \\
Linear Regression & 1 & $7.701^{* *}$ & $5.653^{*}$ & $12.946 * *$ & $10.360 * *$ \\
Deviations & 1 & 0.067 & 4.182 & 3.104 & 0.554 \\
Within Cells (MS) & 27 & $(0.061)$ & $(0.072)$ & $(0.074)$ & $(0.092)$ \\
Total (Sum of Squares) & 29 & $(2.146)$ & $(2.651)$ & $(3.186)$ & $(3.491)$ \\
\hline * $p<.05, * * p<.001$ & & & & & \\
\hline
\end{tabular}

response data of each $S$ were arranged into matrix form, and from each of the 240 matrices the amount of information transmitted, or $\mathrm{H}(\mathrm{t})$, was computed with use of the appropriate formula based on response information and equivocation (see Garner \& Hake; 1951, p. 449). This was then divided by S's median RT to provide an estimate of each S's rate of information transmission, or $\mathrm{H}(\mathrm{t}) / \mathrm{RT}$, in bits/sec. The mean rates of information transmission are shown in Fig. 3 as a function of stimulus dissimilarity for each of the four levels of irrelevant information employed.

An analysis of variance of the data of Fig. 3 indicated that there were statistically significant differences among the six levels of stimulus dissimilarity $(F=$ 110.936, df $=5 / 216, p<.001$ ), the four levels of irrelevant information $(F=15.713$, $\mathrm{df}=3 / 216, \mathrm{p}<.001)$, and the interaction of the two variables $(F=2.428, \mathrm{df}=$ $15 / 216, p<.005)$. The interaction appears to be due principally to the differences between the function obtained at the 0 -bits/stimulus level of irrelevant information and the other three functions. This interpretation is further supported by the results of a trend analysis suggested by the previous finding of a logarithmic relation between $\mathrm{H}(\mathrm{t}) / \mathrm{RT}$ and $\mathrm{SD}$ value (Thurmond \& Alluisi, 1963, pp. 335-336). In the present case, the overall (logarithmic) trend was statistically significant $(F=539.774, \mathrm{df}=1 / 216, \mathrm{p}<.001)$, but so also were the deviations from regression $(F=3.725$,

Table 2. F-Ratios for Variances Due to Linear (Log) Regression and Deviation From Regression at Each of Four Levels of Irrelevant Information

\begin{tabular}{lrrrrr} 
& \multicolumn{5}{c}{ Irrelevant-Information Conditions } \\
$\begin{array}{l}\text { Source of } \\
\text { Variation }\end{array}$ & df & 0 & 1 & 2 & 3 \\
\hline Linear(Log) Regression & 1 & $53.740 * *$ & $155.382 * *$ & $19.657^{* *}$ & $295.040 * *$ \\
Deviations & 4 & $2.650 *$ & 2.303 & 0.513 & 0.794 \\
Within Cells (MS) & 54 & $(0.099)$ & $(0.094)$ & $(0.150)$ & $(0.068)$ \\
Total (Sum of Squares) & $59(11.749)$ & $(20.590)$ & $(26.410)$ & $(23.918)$ \\
\hline$* p<.05 ; * * p<.001$ & & & & &
\end{tabular}

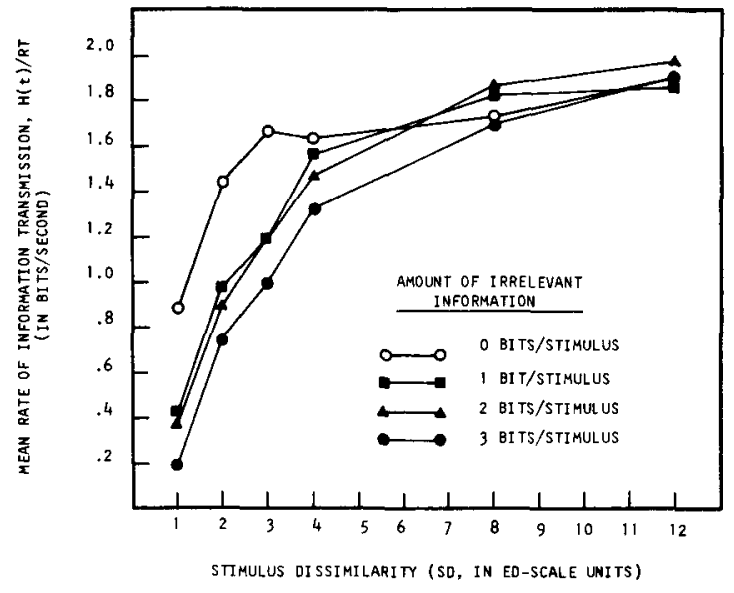

Fig. 3. Rate of information transmission as a function of stimulus dissimilarity at four levels of irrelevant information.

$\mathrm{df}=4 / 216, \mathrm{p}<.01)$. The major deviations appear to have been contributed by the 0 -bits/stimulus level of irrelevant information.

These analyses of variance were extended, and four regression analyses were computed-one at each of the different levels of irrelevant information. The results of these analyses are summarized in Table 2. It is apparent that the logarithmic trend was statistically significant at each of the four levels of irrelevant information, and that the deviations from regression were significant only at the 0 -bits/stimulus level. The slope constant of the 0 -bits/stimulus level of irrelevant information differed significantly from those of the other three levels $(t=3.291,3.821,5.143$, respectively, $d f=116, p<.001$ in each case) whereas none of the remaining differences between slope constants was statistically significant.

\section{Discussion}

At each of the four levels of irrelevant information used in the present study, the functional relation obtained between each of the three measures of performance (errors, $\mathrm{RT}$, and $\mathrm{H}(\mathrm{t}) / \mathrm{sec}$.) and stimulus dissimilarity (or SD value) was essentially identical in form to that previously obtained with no irrelevant information (see Thurmond \& Alluisi, 1963). Thus, in both studies, errors and RTs were generally found to be different for the LD and HD subgroupings; i.e., they were found to be disjunctive about the critical SD value of 3.5 units. Also, in three of the four cases here, $\mathrm{H}(\mathrm{t}) / \mathrm{sec}$. was found to be a single (logarithmic) function of stimulus dissimilarity; in the fourth case, at the 0-bits/stimulus level of irrelevant information, the deviations from regression were statistically significant. However, since this is the one condition that replicated the previous study in which the deviations were found to be nonsignificant (cf., Thurmond \& Alluisi, 1963, pp. 335-336), the significance of the deviations found in the present study 
can be interpreted cautiously only as evidence of a type-I error.

Thus, it appears that the presence of up to 3 bits/ stimulus of irrelevant information will not affect the forms of the functions obtained. Irrelevant information does not change the nature of the task nor the nature of S's behavior. It does, however, change the levels at which $S$ is able to perform, especially where difficulties of discrimination are involved.

For example, the data of Fig. 1 indicate, in terms of errors, that the presence of increasing amounts of irrelevant information has increasingly detrimental effects on S's performance at SD values below 3.5 (the LD subgroupings that confound stimulus dissimilarity and discriminability), but essentially no effect at $\mathrm{SD}$ values above 3.5 (the HD subgroupings that represent differences in stimulus dissimilarity at a more-or-less uniformly high level of discriminability). Furthermore, the significant interaction of these two variables seems to suggest that the detrimental effects of irrelevant information on the accuracy of absolute judgments will be disproportionally greater as difficulties of discrimination are increased (i.e., as SD values are lowered).

No such interaction between stimulus dissimilarity and irrelevant information was exhibited in the RT data (cf., Fig. 2 and Table 1), although the LD and HD subgroupings differed in slope under two of the four irrelevant-information conditions. The direction of these differences also supports the observation that the detrimental effect of irrelevant information tends to increase with increases in the difficulty of discrimination.

Since the different Ss may have traded-off speed and accuracy differently in the different conditions, the $H(t) / R T$ data that combine the speed and accuracy aspects of performance provide what is, perhaps, the clearest indication of the combined effects of stimulus dissimilarity and irrelevant information. $\mathbf{H}(\mathrm{t}) / \mathbf{R T}$ appears to be a negatively accelerated increasing function of stimulus dissimilarity at each of the four levels of irrelevant information (cf., Fig. 3 and Table 2). Although the overall rate of information transmission is detrimentally affected by increases in the amount of irrelevant information, the effects at the lower SD values (where the discriminations are difficult) are greater than at the higher values (where the discriminations are easy).

As was indicated in the introduction, several prior investigations have demonstrated that irrelevant information has a detrimental effect on performance (Archer et al, 1954; Gregg, 1954; Hodge, 1959; Montague, 1965), whereas other investigations have failed to demonstrate any such effect (Archer, 1954; Morin et al, 1961). One of the pertinent factors that determines whether irrelevant information will or will not affect performance is the likelihood that the information will or will not give rise to competing responses; as Hodge (1959) has shown, performance will be affected detrimentally when the irrelevant information is highly likely to stimulate competing responses, as when it is "sometimes relevant."

The results of the present study serve to identify an additional variable that influences the effects of irrelevant information on absolute judgments; namely, the difficulty of the discrimination required. In general, the more difficult the discrimination, the greater the detrimental effect of irrelevant information on performance. This is clearly true with respect to accuracy and the rate of information transmission. The results are less clear with respect to speed, but a similar relation is indicated. At any rate, these results suggest that valid interpretations of the effects of irrelevant information can be made only in conjunction with clear assessments of the difficulties of the discriminations required.

\section{References}

Alluisi, E. A., \& Sidorsky, R. C. The empirical validity of equal discriminability scaling. J. exp. Psychol., 1958, 55, 86-95.

Archer, E. J. Identification of visual patterns as a function of information load. J. exp. Psychol., 1954, 48, 313-317.

Archer, E. J., Boume, L. E., Jr., \& Brown, F. G. Concept identification as a function of irrelevant information and instructions. J. exp. Psychol., 1955, 49, 153-164.

Garner, W. R., \& Hake, H. W. The amount of information in absolute judgments. Psychol. Rev., 1951, 58, 446-459.

Gregg, L. W. The effect of stimulus complexity on discriminative responses. J. exp. Psychol., 1954, 48, 289-297.

Hodge, $M$. $\boldsymbol{H}$. The influence of irrelevant information upon complex visual discriminations. $J$. exp. Psychol, 1959, 57, 1-5.

Montague, W. E. Effect of irrelevant information on a complex auditory discrimination task. $J$. exp. Psychol., 1965, 69, 230-236.

Morgan, B. B., Jr. The effect of irrelevant information on absolute judgments at different levels of discrimination difficulty. Unpublished Master's Thesis, University of Louisville, 1965.

Morin, R. E., Forrin, B., \& Archer, W. Information processing behavior: the role of irrelevant stimulus information. J. exp. Psychol., 1961, 61, 89-96.

Muller, P. F., Jr., Sidorsky, R. C., Slivinski, A. J., Alluisi, E. A., $\&$ Fitts, P. M. The symbolic coding of information on cathode ray tubes and similar displays. USAF WADC Tech. Rep., 1955, No. 55-375.

Thurmond, J. B., \& Alluisi, E. A. Choice time as a function of stimulus dissimilarity and discriminability. Canad. J. Psychol., $1963,17,326-337$.

\section{Note}

1. Supported in part by the U. S. Army Medical Research and Development Command, Department of the Army, under Contract No. DA-49-193-MD-2567, "Behavioral Effects of Infectious Diseases." This paper is based on a master's thesis submitted by the senior author at the University of Louisville; the thesis was prepared under the direction of the junior author. An abridged report of this research was presented by the senior author at the 58th annual meeting of the Southern Society for Philosophy and Psychology, April 1966, New Orleans La.

(Accepted for publication, November 20, 1966.) 